\title{
Effects of Coupling and Phase Imperfections in Programmable Photonic Hexagonal Waveguide Meshes
}

\author{
IMAN ZAND ${ }^{1,2}$ AND WIM BOGAERTS ${ }^{1,2, *}$ \\ ${ }^{1}$ Ghent University - IMEC, Photonics Research Group, Department of Information Technology. Technologiepark-Zwijnaarde 126, 9052 Ghent, Belgium. \\ ${ }^{2}$ Center of Nano and Biophotonics, Ghent University, Ghent, Belgium. \\ ${ }^{*}$ Corresponding author: wim.bogaerts@ugent.be
}

Compiled November 25, 2019

\begin{abstract}
We present a study of the effect of the imperfections on the transmission and crosstalk in programmable photonic mesh circuits consisting of tunable couplers and phase shifters. The many elements in such a mesh can generate a multitude of parasitic paths when the couplers and phase shifters deviate even slightly from their nominal value. Performing Monte-Carlo simulations, we show that small stochastic imperfections in the phase and coupling $(<1.0 \%)$ can introduce unwanted interferences and resonances and significantly deteriorate the frequency response of the circuit. We also demonstrate that in the presence of imperfections the programming strategy of the unused couplers can reduce effects of such parasitics.

(C) 2019 Optical Society of America
\end{abstract}

http://dx.doi.org/10.1364/ao.XX.XXXXXX

\section{INTRODUCTION}

Recent developments in photonic integrated circuits (PICs) technologies have enabled a wide range of research on programmable PICs for various applications such as optical communication, artificial neural networks, and quantum information technologies[1-15]. Broadband switches [1], microwave photonic subsystems [6], and universal multiport photonic interferometers (important for advanced optical functions) $[3,11,12,14,16-18]$ are examples of recent demonstrations in this field. It is hoped that, similar to the field programmable gate arrays (FPGAs) in electronics, multi-functionality of programmable photonic circuits will enable us to considerably reduce required time and cost to design, fabricate, and test new photonic chips.

In most programmable PIC architectures, the optical signal processing tasks take place in a linear optical core consisting of a waveguide mesh where the topology and connectivity defines the possible implementable functionalities. These meshes are normally constructed using tunable optical building blocks such as couplers/beam splitters and phase shifters, controlled by electronics. We can distinguish two main approaches to implement such meshes: 'forward-only meshes' $[3,9,11,12,15,16]$ and 'meshes with feedback loops' [2, 10, 14, 17, 19-22]. In the first approach, linear optical transformations are implemented by one-way propagation from a set of input ports to a set of output ports, essentially implementing a transmission matrix (T-matrix).
In most implementations, all optical paths have the same length (apart from a $0-2 \pi$ phase shift), and the response is largely wavelength independent.

In the second type of meshes, the waveguides are connected in loops that can implement differential delay lines, ring resonators, and optical feed-back circuits, enabling applications such as finite/infinite impulse response (FIR/IIR) multiport interferometers and RF filters [21]. In this approach, all ports can serve as inputs and outputs, and the linear optical operation is described by a wavelength dependent scatter matrix (S-matrix). These two-dimensional (2D) waveguide meshes/lattices have much higher flexibility $[2,10,14,17,19-22]$, as they can also be configured to function as a forward-only mesh. The first demonstration of integrated waveguide meshes, which had been inspired by FPGAs, was done by Zhaung et.al. using square lattices and Mach-Zhender Interferometers (MZIs) [2]. Later, triangular and hexagonal lattices were studied by Pérez et al. [19]; they showed that hexagonal meshes are superior in terms of various figures of merit such as spatial tuning reconfiguration step and reconfiguration performance.

The physical implementations of such programmable photonic circuits consist of many identical optical building blocks (phase shifters and tunable couplers), and the routing of the light is controlled by electronics and software. Such a chip, therefore, requires electronic drivers for all the optical elements, an assembly scheme for fibers and high-speed connections, and algorithms and software that will allow a designer to actually 
implement a useful function. Hence, there are various possible sources of errors, both in design and fabrication, causing imperfect behavior of the optical building blocks, which in turn will introduce parasitic behavior in the mesh. These errors (acting as additional loss, phase errors, and coupling errors) can be accumulated and dramatically deteriorate the response of the circuit $[18,22]$. In particular, for the meshes with feed-back loops, they create a multitude of secondary and tertiary paths for the light, which can cause unwanted interferences and resonances, and thus affect the frequency response of circuit [22]. This will result in a wavelength dependent transmission 'ripple' in the desired output ports, and crosstalk in the other ports.

We can discern three types of parasitics originating from the phase shifters and tunable couplers:

- Nonidealities in the optical transmission where light remains in the intended waveguide paths. This can be an error in the phase shift, or an error in the coupling ratio. These, in principle, could be compensated with improved control electronics and algorithms of the tunable building blocks.

- Nonidealities in the components where light is coupled to a waveguide path where it should never end up in during normal operation. The most common example is backscattering in waveguides, and backreflection/backcoupling in tunable couplers [23].

- Nonidealities that radiate light from the waveguides altogether. Due to reciprocity, such defects can also capture light radiated elsewhere on the chip, which can introduce secondary light paths.

In this paper, we focus on the first type of nonidealities and study the effect of small stochastic imperfections in the phase and coupling control of a 7-cell hexagonal mesh consisting of $2 \times 2$ couplers and zero-length phase shifters connected by waveguides. For the simulations, we have extended the photonic circuit simulator Caphe (part of the design framework IPKISS) [24] with models for the mesh components, and performed Monte-Carlo simulations to evaluate the effect of imperfections.

\section{SIMULATION METHODOLOGY}

As a baseline case for the simulations, we have constructed a uniform mesh based on hexagonal cells which offer flexible programming, especially since this mesh type allows clockwise/counterclockwise coupling [19]. Figure 1 shows a schematic representation of a hexagonal mesh consisting of 7 hexagonal cells. As seen, the arms of the $2 \times 2$ couplers (CPs) are connected to the zero-length phase shifters (PSs) through silicon waveguides. We also assume that a perfect control over the variable property of each component is feasible, and this is over its entire operational range. This limit is $0-100 \%$ coupling efficiency for the CPs and $0-360^{\circ}$ phase delay for the PSs. We also assume that all elements can be controlled independently, ignoring at this stage effects of tuning crosstalk (e.g. through thermal crosstalk). The total length of each segment is assumed to be $300 \mu \mathrm{m}$ with a waveguide bend radius of $10 \mu \mathrm{m}$.

Our circuit generator is implemented using Python on top of the IPKISS/Caphe framework by Luceda Photonics, which generates hexagonal lattices with arbitrary configurations of the cells. Mesh components are implemented as parametric cells in the IPKISS framework, so they contain a layout, connectivity
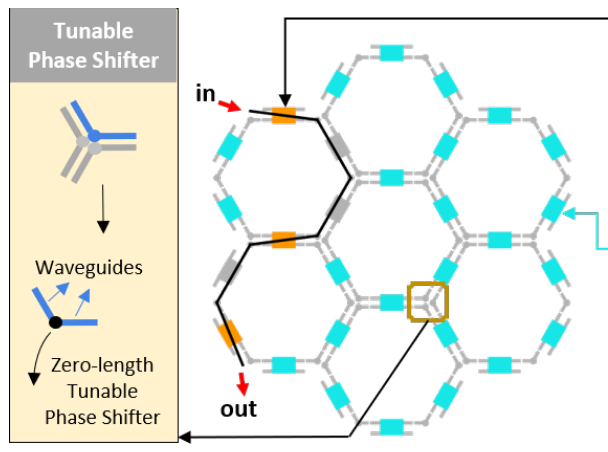

Routing Couplers

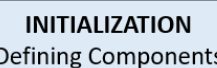

\& Circuit Parameters

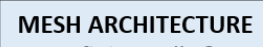

Defining Cells \& Configuring Mesh
Caphe Simulator
Extracting S-Matrix \& Frequency Sweep
Data Analysis

Fig. 1. Schematic representation of the 7-cells hexagonal mesh and the simulation workflow. For each mesh configuration, there are two types of couplers: Routing Couplers (involved in defining light paths) and Unused Couplers (their state, in principle, does not affect the light paths). In Normally Bar bias (NB bias), unused couplers are programmed in the bar state, while, in Normally Cross bias (NC bias), they are programmed in the cross state.

information (netlist), and a circuit model. This means that both realistic and abstract building blocks of the components can be used for the calculations and the generated layouts can be used for the fabrication. Also, as the layout feeds back into the circuit simulation, actual waveguide lengths and device parameters are being used. Even though the components are considered identical in the entire mesh, we can set the circuit model parameters for each instance individually to 'program' the circuit. In the Caphe circuit simulator, the optical properties such as phase shift (for the PS blocks), coupling coefficient (for the CP blocks), and insertion loss (for both) can be varied. We can also visualize the model parameters for each building block as part of the overall circuit. This can be used to test programmability, but also to evaluate sensitivity of the circuit to variations.

In order to evaluate the response of the circuit subject to parasitic imperfections, we consider deviations from the assigned values for the phase delay of the PSs and the coupling coefficient of the CPs. Otherwise, the rest of the properties are assumed to be unperturbed. The errors are described as a normal distribution around the parameters' nominal values with varied standard deviations (hereafter $\sigma$ ) is considered. In the MonteCarlo simulations, a population of 100 experiments is defined for which the parameter values are randomly generated.

\section{RESULTS AND DISCUSSIONS}

In a programmable photonic circuit, routing is the most prevalent functionality to be implemented, as it is used to interconnect all other functions. For simple routing, couplers will only be configured in either cross (coupling coefficient $\kappa=1$ ) or bar state $(\kappa=0)$. In contrast with ordinary circuits, where routing is done through static waveguides, in a programmable circuit the routing is done by the tunable couplers. As a result, there are many more possibilities for light to leak into adjacent paths and 
cause undesired interference within the circuit.

The couplers which are used to create the actual route are referred as Routing Couplers (RCPs) and remained couplers in the mesh are the Unused Couplers (UCPs); UCPs are not involved in the routing and under perfect operation conditions, their state does not affect the path shape. However, as we will see further, their state does affect the function of the circuit in the presence of imperfections. Therefore, we define two different biasing schemes: setting all the UCPs in 1) bar state or 2) cross state. We refer to these biases as "Normally Bar" (NB) and "Normally Cross" (NC), respectively (Fig. 1). For the schematic demonstration of the unused and routing couplers, we have chosen the blue color for the UCPs and gray/orange color for the RCPs when they are in the bar/cross state, respectively.

\section{A. Single Paths}

Figure. 2a shows a path ( with $L_{\text {path }}=6 L_{u}$ ) routed through a 7-cells mesh, and Fig. $2 b$ plots the transmission from the input to the output port for NB bias (red lines) and NC bias (green lines), with random variations in the couplings $\kappa$ with $\sigma_{k}=0.05 \%, 0.4 \%, 1.0 \%$ and variations in the phase delays $\Phi$ with $\sigma_{\phi}=17^{\circ}$. When the mesh is ideally programmed (black dashed lines), we expect that the length of the path will only contribute losses. But as the graph for the NB bias shows, when random coupling variations increases, we see that levels of transmissions drop and significant ripples appear on the output spectrum. In fact, unwanted coupling will introduce additional losses because light is tapped out of the main path, and, also, it introduces par-

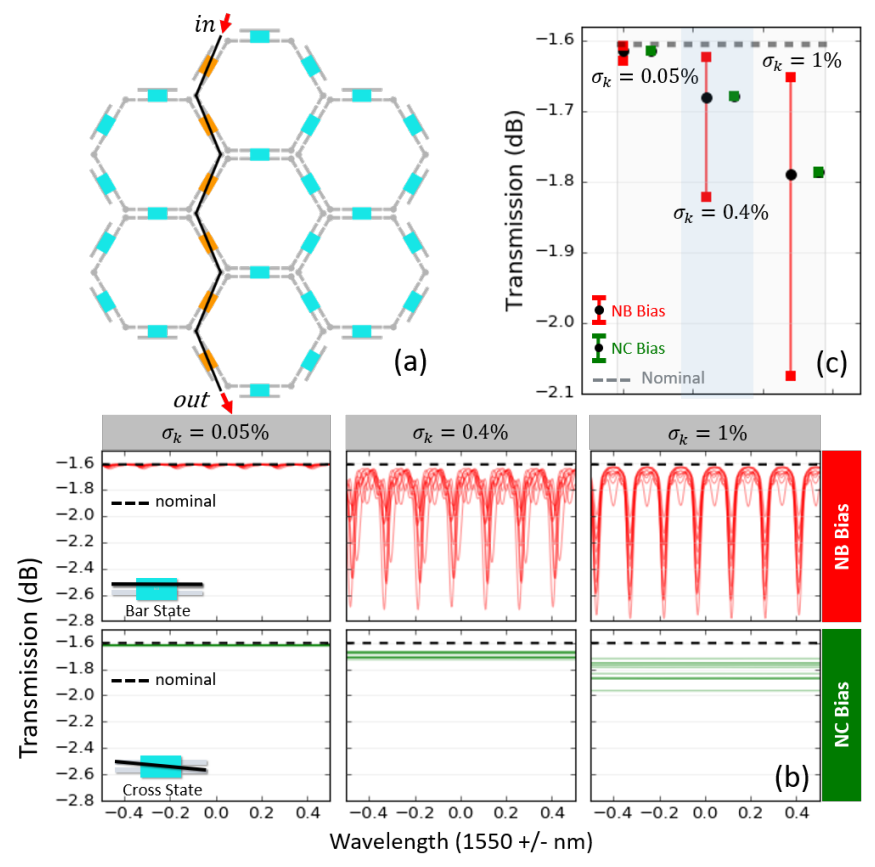

Fig. 2. a) Schematic of a routed path (with $L_{\text {path }}=10 L_{u}$ ) within a 7-cells mesh b) Transmission spectra of the mesh for two types of biasing: Normally-Bar (NB): unused couplers are biased in the bar state (red curves) and Normally-Cross (NC): unused couplers are biased in the cross state (green curves). The results are plotted for $\sigma_{k}=0.05 \%, 0.4 \%, 1.0 \%$ from left to right. c) Intensity spread analysis of the transmission in the output for random variations of $\sigma_{k}=0.05 \%, 0.4 \%, 1.0 \%$. Red and green error bars correspond to the NB and NC biases. asitic interference paths and even ring resonators. Although additional losses caused by unwanted couplings from the main path are inevitable, it is expected that proper programming of the unused couplers (using NC bias) suppresses parasitic interference paths and prevent ripples of the transmission response. As shown in Fig. 2b, using NC bias can successfully compensate light accumulation in the mesh and suppress the formation of the coupled ring resonators in the mesh. As a result, the ripples of transmission have been disappeared.

To analyze the response of the programmed mesh for the NB and NC biases, we collected the $[5 \%-95 \%]$ intensity spread in the transmission spectra of Fig. $2 b$ for 100 Monte-Carlo simulations with the different random coupling variations of $\sigma_{\mathcal{K}}=0.05 \%, 0.4 \%, 1.0 \%$ and plotted them as red (NB bias) and green (NC bias) error bars in Fig. 2c. The [min, mean, max] points of the error bars are obtained by:

$$
\begin{array}{r}
\min =\min \left(\left[\min (T(\lambda))_{5}, \ldots, \min (T(\lambda))_{95}\right]\right) \\
\text { mean }=\operatorname{mean}\left(\left[\operatorname{mean}(T(\lambda))_{5}, \ldots, \operatorname{mean}(T(\lambda))_{95}\right]\right) \\
\max =\max \left(\left[\max (T(\lambda))_{5}, \ldots, \max (T(\lambda))_{95}\right]\right)
\end{array}
$$

The subscript 5 and 95 indicate that we only considered the $5^{\text {th }}-95^{\text {th }}$ percentile of the samples, discarding the most extreme values. Comparing error bars clearly shows that NC bias considerably reduces the intensity spread of the transmission ( $>95 \%$ ).

We performed this intensity spread analysis on a variety of both simple and complex paths for NB and NC biases (Fig. 3). Similar to Fig. 2c, error bars are plotted for 3 different values of $\sigma_{k}$ and compared with the nominal response of the path (shown by black dashed lines). Each configuration is illustrated either above or below its corresponding intensity spread error bars. As seen, for NB bias, larger $\sigma_{k}$ causes more intensity spread (larger ripples in spectrum) and losses, and complex paths with loop in their configuration ( $\mathrm{L}, \mathrm{M}, \mathrm{N})$ have error bars with a maximum transmission higher than the nominal values; in fact, effects of parasitic shortcuts (shorter paths with lower loss than the main path) are more prominent and can result in constructive interference of the output signal arriving through shortcuts. On the other side, for the NC bias, the effect of parasitic interference is almost eliminated for the simple paths, and, for the more complex paths, although this benefit diminishes, we still see a reduction of $50 \%$ in the intensity spread compared to NB bias.

\section{B. Multi-Path Routing}

The advantage of a programmable circuit is that we can implement multiple functions at the same time, connect multiple inputs to multiple outputs, and even use the tunable couplers as crossings to make intersecting paths. Of course, when there are imperfections in such scenarios, it is important that crosstalk between different paths is kept to a minimum. Figures. $4 \mathrm{a}$ and $4 \mathrm{~b}$ show the intensity spread of the transmission and crosstalk spectra of a mesh configured for the vertical and horizontal double-paths (A and C) and a multi-paths composed of them (B).

It is clear from Fig. 4 that putting the circuit in NC bias (green bars) eliminates parasitic shortcuts for all the cases and their transmission intensity spread reaches to $0 \mathrm{~dB}$. This means that, similar to Fig. $2 b$, all ports will have an almost flat transmission response without ripples. However, increasing $\sigma_{k}$ will increases losses and reduce transmitted power which is inevitable. In NB bias, effect of parasitics is noticeable for the double-paths (A and $\mathrm{C})$, while it has been reduced for the $\mathrm{C}$ configuration. The reason 


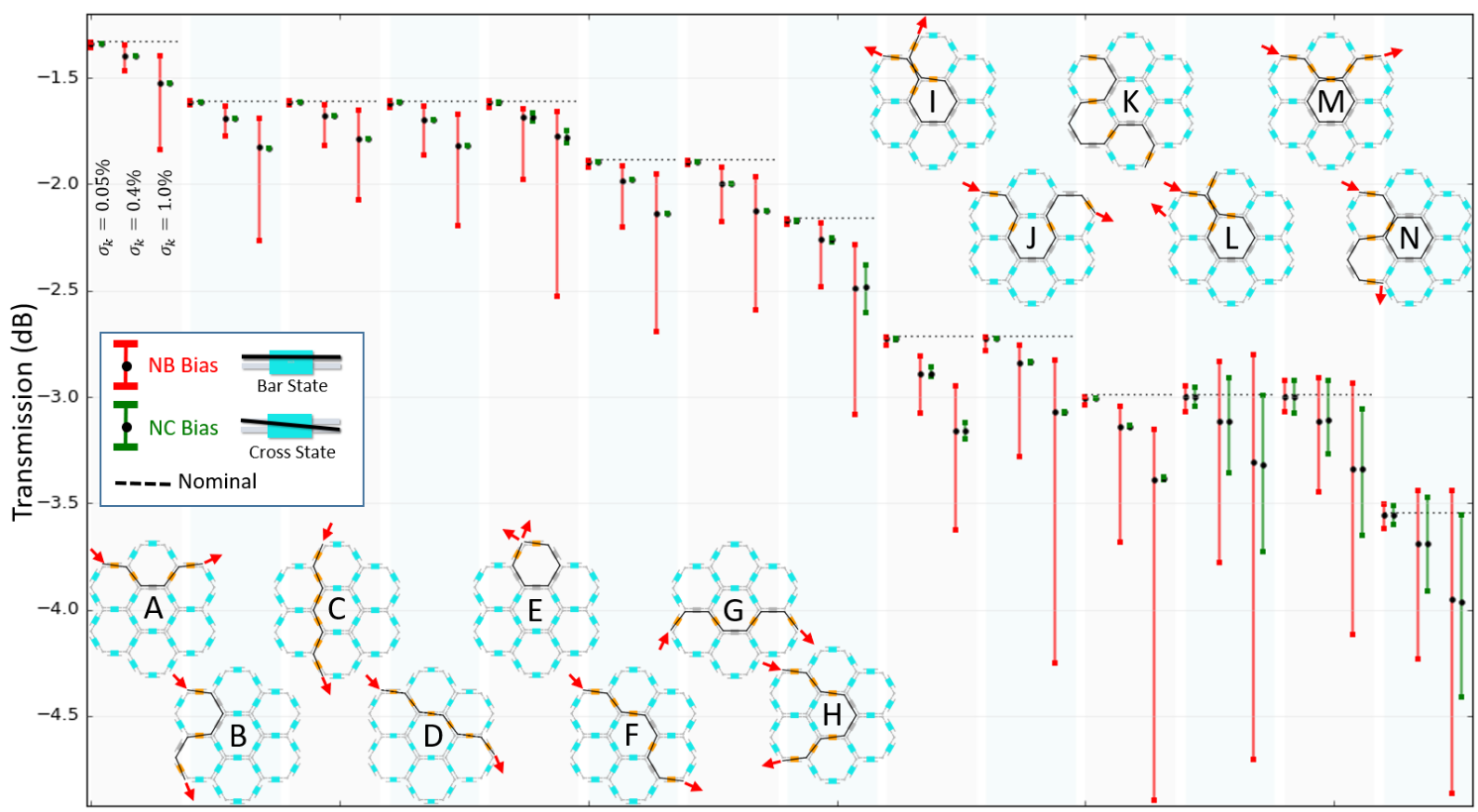

Fig. 3. Intensity spread analysis of different configurations of the 7-cells mesh to study both simple and complex paths. Blue couplers are in the Bar (Cross) state for the NB (NC) bias. Random variations of $\sigma_{k}=0.05 \%, 0.4 \%, 1.0 \%$ are chosen for the Monte-Carlo simulations. Red and green error bars correspond to the NB and NC bias, respectively.

is that the C configuration has more couplers in cross state, which breaks loops in the mesh and stops light accumulation inside of it. For the crosstalk, plotted in Fig. $4 \mathrm{~b}$, increasing $\sigma_{k}$ increases its value for all configurations for both NB and NC biases. It is also seen that outputs in $\mathrm{A}\left(\mathrm{O}_{1}\right.$ and $\left.\mathrm{O}_{2}\right)$ and $\mathrm{C}\left(\mathrm{O}_{3}\right.$ and $\mathrm{O}_{4}$ ) configurations have higher crosstalk compared to the $\mathrm{B}$ configuration.

\section{Mach-Zehnder interferometers}

Mach-Zehnder interferometers (MZI) are the basis of all finiteimpulse-response filters. The performance of a single MZI is a measure of how easily and accurately higher-order filters can be implemented. A simple MZI can also be easily evaluated for imperfections from the point of view of extinction ratio and absolute wavelength registration.

Figure. 5a shows 3 different configured MZIs (A: $\Delta L=6 L_{u}$, B: $\left.\Delta L=4 L_{u}, \mathrm{C}: \Delta L=10 L_{u}\right)$. The corresponding transmission responses of these three configurations are plotted in Fig. 5b, where we have again used red and green curves for NB and $\mathrm{NC}$ biases. Here, to show the effect of NB and NC biases, 10 cycles of Monte-Carlo simulations are used for $\sigma_{k}=1 \%$ and $\sigma_{\phi}=17^{\circ}$. Also, for simplicity, only one of the outputs $\left(O_{1}\right)$ has been shown (the other one, $\mathrm{O}_{2}$, has similar behavior). As seen, while the coupling errors mainly cause deterioration in the extinction ratio and weak appearance of other harmonics, the error in the phase shifters introduces a redshift or blueshift in the spectrum. Although phase shifter errors deteriorate the responses for NC and NB biases, NC bias shows better extinction ratios.

In order to quantify performance of the circuit for the selected MZI configurations, a correlation-based analysis has been performed using 100 cycles of Monte-Carlo simulations and the results are shown in Fig. 5c. Here, three pairs of coupling and phase variations $\left(\sigma_{k}, \sigma_{\phi}\right)$ have been selected: $\left(0.05 \%, 17^{\circ}\right)$, $\left(1.0 \%, 17^{\circ}\right)$, and $\left(1.0 \%, 2^{\circ}\right)$. For this analysis, the nominal response without perturbation is considered as the reference signal $(R)$, and, for the perturbed responses $(S)$, the correlation with the $R\left(\operatorname{Corr}(S, R)^{2}\right)$ is calculated. The auto-correlation of the reference $\left(\operatorname{Corr}(R, R)^{2}\right)$ has its maximum at the zero shift $\left(\left.\operatorname{Corr}(R, R)^{2}\right|_{\Delta \lambda=0}\right)$ and both correlation functions show small local maxima due to their periodic behaviour. The closer the period of the two signals, the more similar these local maxima. The correlation graphs are also normalized with respect to the auto-correlation of $S\left(\operatorname{Corr}(S, S)^{2}\right)$ and $R\left(\operatorname{Corr}(R, R)^{2}\right)$. In other words, the normalized correlation function can be read as:

$$
\widetilde{\operatorname{Corr}}(S, R)^{2}=\frac{\operatorname{Corr}(S, R)^{2}}{\sqrt{\operatorname{Corr}(S, S)^{2}\left|\max * \operatorname{Corr}(R, R)^{2}\right|_{\max }}}
$$

The value $\left.\widetilde{\operatorname{Corr}}(S, R)^{2}\right|_{\Delta \lambda=0}$ corresponding to the normalized correlation of $S$ with $R$ is an indicative value of the resemblance of the nominal and perturbed responses. At a shift wavelength of $\Delta \lambda_{\mathrm{MAX}}$ the normalized correlation curve of $S$ has a maximum $\left.\widetilde{\operatorname{Corr}}(S, R)^{2}\right|_{\text {MAX }}$. This indicates that in average, $S$ has a $\Delta \lambda_{\mathrm{MAX}}$ shift as compared to $R$, and if such a shift was absent (or unimportant depending on the application), the resemblance value of $S$ and $R$ would be $\left.\widetilde{\operatorname{Corr}}(S, R)^{2}\right|_{\text {MAX }}$. Figure. 5c shows calculated

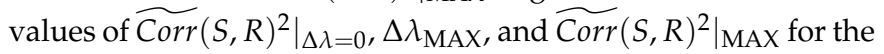
configured MZIs and different pairs of $\left(\sigma_{k}, \sigma_{\phi}\right)$, where we have used error bars to compare NB (red) and NC (green) biases. As seen, phase error, compared to coupling error, is the prominent factor and by reducing it to $2^{\circ}$ we can achieve acceptable resemblance with the nominal response. It is also seen that $\mathrm{NC}$ bias, unlike with the basic routing, does not improve the results for the MZI configurations. 


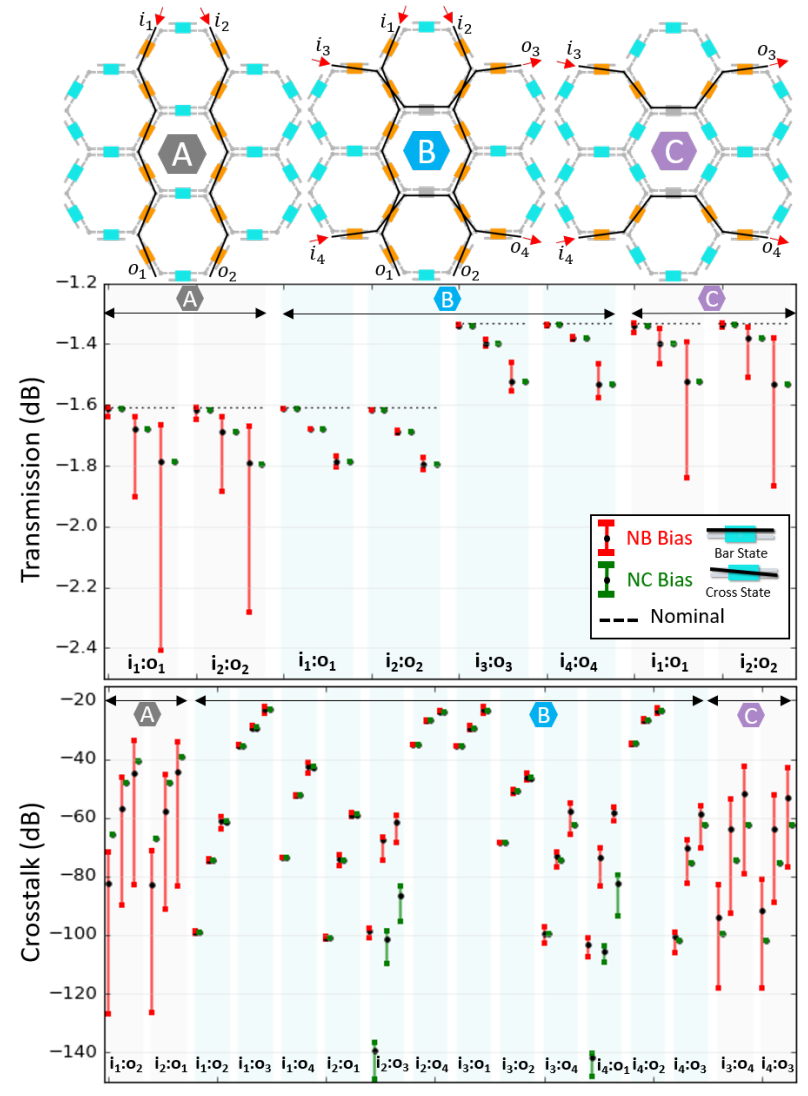

Fig. 4. Intensity spread analysis of the a) Transmission and b) Crosstalk of double- and multi-paths. Similar to the Fig. 2c, error bars are plotted for $\sigma_{k}=0.05 \%, 0.4 \%, 1.0 \%$.

\section{Ring Resonators}

Ring resonators which are the building blocks of many silicon photonics filters are susceptible to peak-splitting due to backreflection. While we did not incorporate backreflection in the parasitic analysis (they are considered as a second type of parasitic), the hexagonal mesh allows for clockwise/counterclockwise coupling through the coupling between two adjacent rings which are not originally designed to share any signal. This has a similar effect as backreflection or backcoupling in the tunable couplers. Figure. 6a shows three configured ring resonators (A, B, C) in our chosen 7-cells hexagonal mesh. For the A and C configurations, rings are located in the center of the mesh; however, their bus waveguides are routed differently. For the B configuration, the ring is located near the boundary of the mesh and its bus waveguide is created by a long and complex path. Transmission responses of these configurations for NB (red) and NC (green) biases with $\left(\sigma_{k}=1 \%, \sigma_{\phi}=17^{\circ}\right)$ are plotted in Fig. $6 \mathrm{~b}$, where 10 cycles of Monte-Carlo simulations have been used. As expected, the phase errors mainly have caused wavelength shifts for all configurations and the coupling error affects the extinction ratio. However, complexity of the path in configuration B has created larger extinction ratio.

We also have analyzed the performance of the ring resonators using the same correlation technique used for the MZIs analysis. Figure. $6 \mathrm{c}$ shows calculated values of $\left.\widetilde{\operatorname{Corr}}(S, R)^{2}\right|_{\Delta \lambda=0}, \Delta \lambda_{\mathrm{MAX}}$, and $\left.\widetilde{\operatorname{Corr}}(S, R)^{2}\right|_{\text {MAX }}$ for the configured resonators and different pairs of $\left(\sigma_{k}, \sigma_{\phi}\right)$. Similar to the MZIs, phase errors can considerably change the shape of the circuit response and by reducing $\sigma_{\phi}$
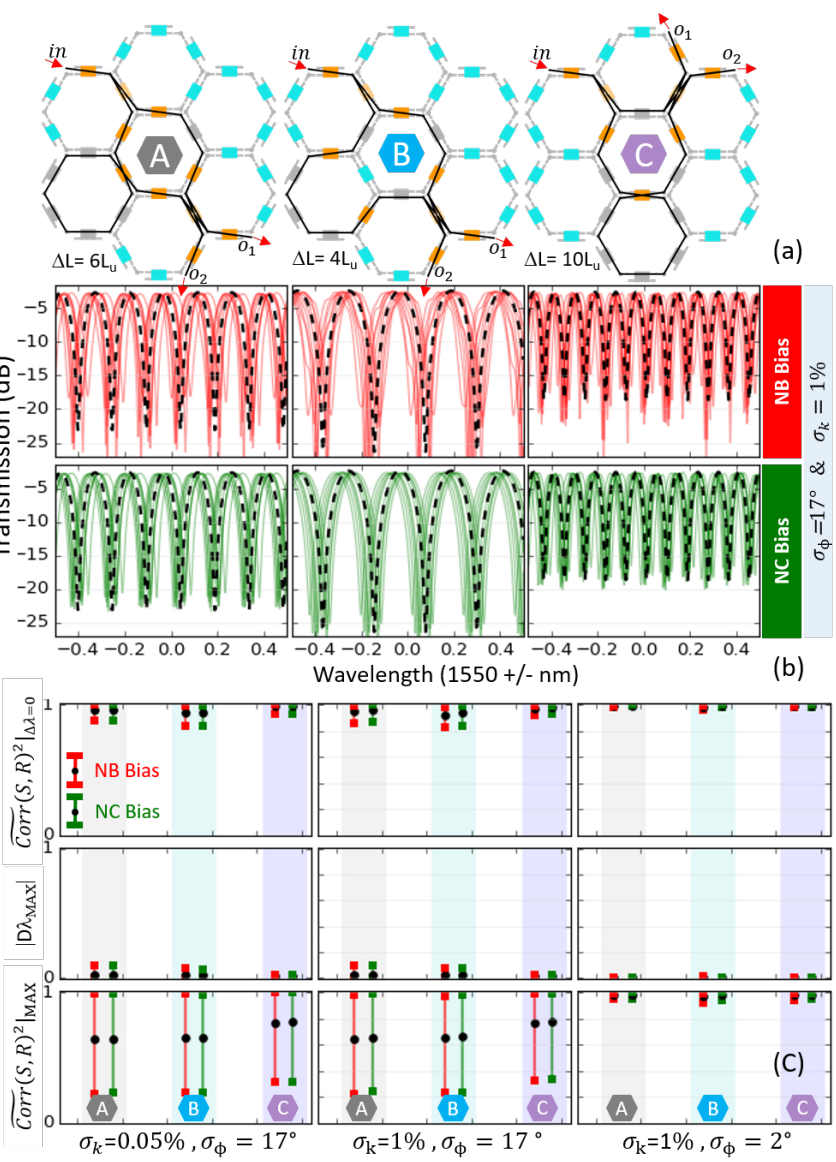

Fig. 5. a) Schematic of the three different configured MZIs in the 7-cells hexagonal mesh (A: $\Delta L=6 L_{u}, \mathrm{~B}: \Delta L=4 L_{u}, \mathrm{C}$ : $\left.\Delta L=10 L_{u}\right)$. b) Transmission response of the MZIs for NB (red) and NC (green) biases, where only 10 cycles of the MonteCarlo simulations have been plotted for better visibility. c) Correlation-based analysis of the configured MZIs for $\left(\sigma_{k}, \sigma_{\phi}\right)$ pairs of $\left(0.05 \%, 17^{\circ}\right),\left(1.0 \%, 17^{\circ}\right)$, and $\left(1.0 \%, 2^{\circ}\right)$.

to $2^{\circ}$ we can achieve a resemblance of more than $90 \%$. Another observation is that although using NC bias can not eliminate effect of phase errors, selecting the ring close to the boundaries (configuration B) can significantly improve performance of the circuit. Comparing configuration $\mathrm{A}$ and $\mathrm{C}$, also, shows that the routing of the path also can effect the response of the circuit even if the rings are in the same location.

\section{E. Splitters}

Another important configuration in programmable circuits are splitters which can be used for multicasting, or as a distribution network for an optical beam former. Here, we present an intensity spread analysis of a $1 \times 4$ (Fig. 7) and $1 \times 16$ (Fig. 8) splitter network. Similar to the previous cases, two biasing schemes (NB and NC biases) are compared and random variations of $\sigma_{k}=0.05 \%, 0.4 \%, 1.0 \%$ and $\sigma_{\phi}=17^{\circ}$ are applied to the couplings $\kappa$ and phase shifts $\Phi$, respectively.

As seen in Fig. 7, similar to the simple routing paths, the coupling error is the dominant factor which affects the power transmission. For NB bias, increasing $\sigma_{k}$ from $0.05 \%$ to $1.0 \%$ results in $0.25 \mathrm{~dB}$ to $1.8 \mathrm{~dB}$ power variation in the transmitted signals to the outputs. Also, higher transmission are seen com- 


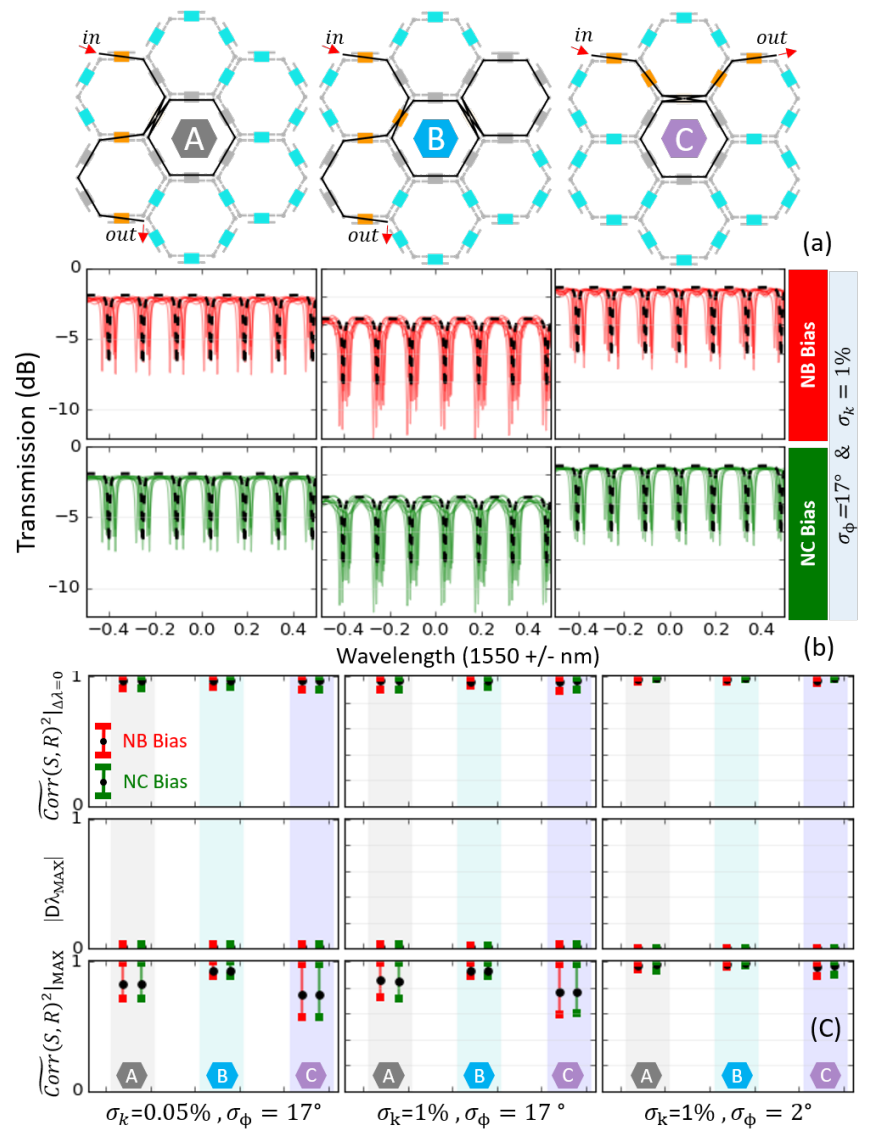

Fig. 6. a) Schematic of three different configured ring resonators in the 7-cells hexagonal mesh. b) Transmission response of the selected configurations for NB (red) and NC (green) biases, where 10 cycles of the Monte-Carlo simulations have been used. c) Correlation-based analysis of the configured ring resonators for $\left(\sigma_{k}, \sigma_{\phi}\right)$ pairs of $\left(0.05 \%, 17^{\circ}\right),(1.0 \%$, $\left.17^{\circ}\right)$, and $\left(1.0 \%, 2^{\circ}\right)$.

pared to their nominal response indicating parasitic shortcuts as discussed for the paths. However, applying NC bias successfully compensates effect of unwanted interferences caused by parasitic couplers and intensity spread reduces more than $95 \%$.

For the $1 \times 16$ splitter, almost all ports and coupler elements of the mesh are actively used and the circuit is operating at its full capacity. Hence, there is a small room to compensate effect of parasitics using unused couplers. This can be observed by comparing green (NC Bias) and red (NB bias) error bars in Fig. 8. As seen, intensity spread reduction by the NC bias is less than $0.2 \mathrm{~dB}$. Also, Similar to the $1 \times 4$ beam splitter, parasitic shortcuts have increased power level of the transmitted signal. Another observation is lower intensity spread of the $\mathrm{O}_{7}-\mathrm{O}_{12}$ ports compared to the others ( $50 \%$ less).

\section{F. Customized Biasing Schemes}

As discussed above, by using NC bias parasitic effects can be eliminated considerably for the paths and splitters. However, setting all of the unused couplers in the cross state will not the only solution for parasitic elimination. In fact, by proper programming of some of unused couplers same or better results can be achieved. To elaborate this, transmission spread analysis of a single path $\left(\Delta L=8 L_{u}\right)$ for the NB bias, NC bias,

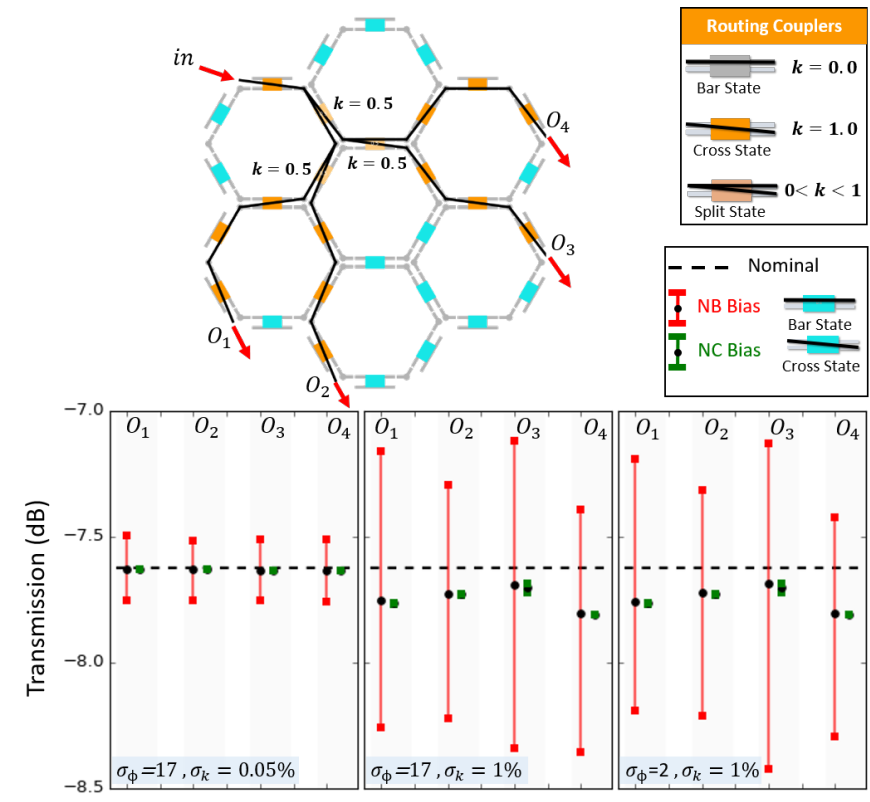

Fig. 7. Intensity spread analysis of a $1 \times 4$ splitter in the 7cell hexagonal mesh. Red and green bars show NB and NC biases, respectively. Similar to Fig. 2c, error bars are plotted for $\sigma_{k}=0.05 \%, 0.4 \%, 1.0 \%$. For NC bias, blue couplers are in the cross state $(k=1)$, while they are in the bar state $(k=0)$ for NB bias.

and three other customized biases are shown in Fig. 9, where $\sigma_{k}=0.05 \%, 0.4 \%, 1.0 \%$. As seen, by programming only few number of unused couplers much better results can be achieved; for the biasing scheme of $\mathrm{E}$, the transmission spread has been reduced by $95 \%$ for $\sigma_{k}=0.1$. The importance of such optimization is in consuming lower energy to eliminate parasitic effects. Hence, for the future studies, optimization algorithms and strategies can be studied in more depth to find the optimum solutions for proper biasing of desired configurations.

\section{CONCLUSION}

Two biasing schemes for the unused couplers in a hexagonal 7-cell mesh are compared for their impact on different configurations of single paths, multi paths, ring resonators, MZIs, and splitters. In these schemes, the unused couplers are set either in Bar state (NB bias) or in the Cross state (NC bias), respectively. Monte-Carlo simulations show that NC bias considerably suppresses the effects of parastics on transmission response of the circuit for paths with different routing. On the other hand, for ring resonators and MZIs in which phase shifters' error is dominant, both biasing schemes have similar effects. It is also shown that by proper programming of few number of unused couplers parasitic effects can be eliminated.

\section{FUNDING}

European Union Horizon 2020 research and innovation programme(780283-MORPHIC) European Research Council (725555-PhotonicSWARM) 

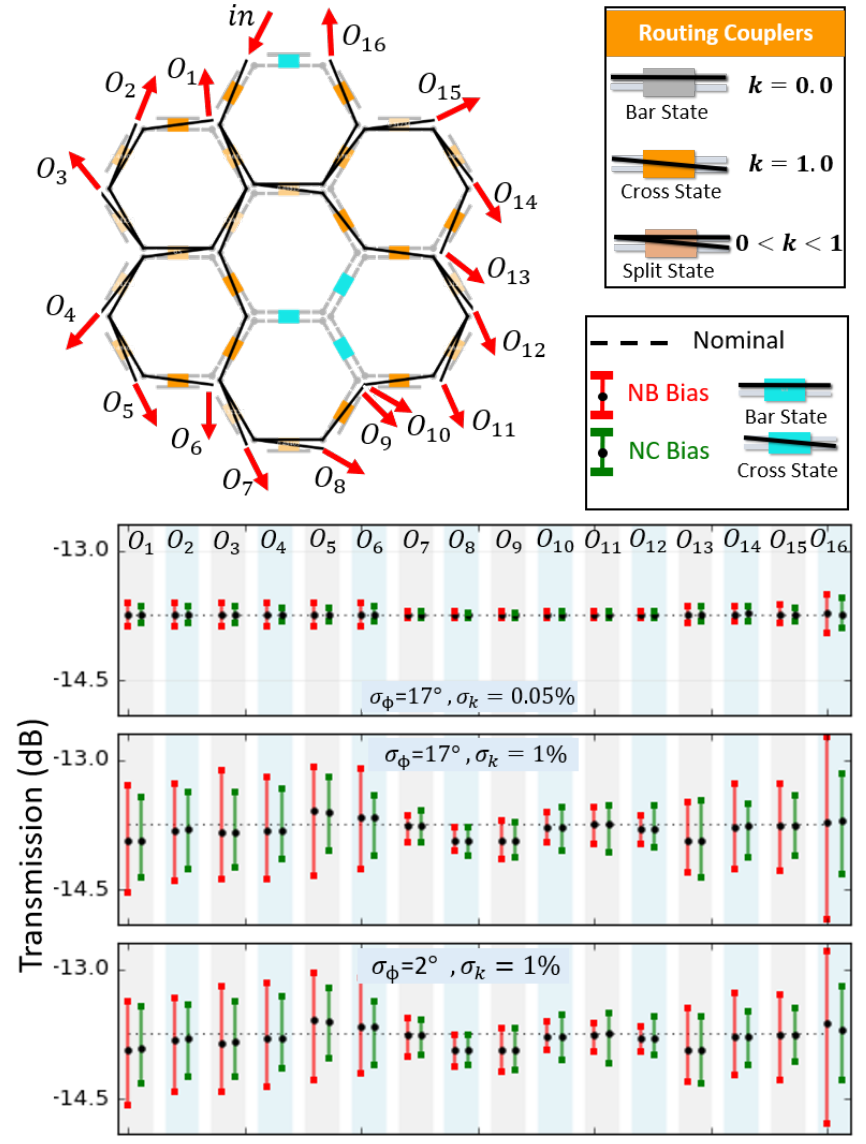

Fig. 8. Intensity spread analysis of a $1 \times 16$ splitter in a 7-cell hexagonal mesh. Red and green bars also show NB and NC biases, respectively. Similar to Fig. 2c, error bars are plotted for $\sigma_{k}=0.05 \%, 0.4 \%, 1.0 \%$. For NC bias, blue couplers are in the cross state $(k=1)$, while they are in the bar state $(k=0)$ for the NB bias.

\section{DISCLOSURES}

The authors declare no conflicts of interest.

\section{REFERENCES}

1. L. Chen, E. Hall, L. Theogarajan, and J. Bowers, "Photonic switching for data center applications," IEEE Photonics J. 3, 834-844 (2011).

2. L. Zhuang, C. G. H. Roeloffzen, M. Hoekman, K.-J. Boller, and A. J. Lowery, "Programmable photonic signal processor chip for radiofrequency applications," Optica. 2, 854 (2015).

3. A. Ribeiro, A. Ruocco, L. Vanacker, and W. Bogaerts, "Demonstration of a $4 \times 4$-port self-configuring universal linear optical component," 2016 Prog. In Electromagn. Res. Symp. PIERS 2016 - Proc. 3, 3372-3375 (2016).

4. D. A. Miller, "Silicon photonics: Meshing optics with applications," Nat. Photonics 11, 403-404 (2017).

5. A. Annoni, E. Guglielmi, M. Carminati, G. Ferrari, M. Sampietro, D. A. Miller, A. Melloni, and F. Morichetti, "Unscrambling light - Automatically undoing strong mixing between modes," Light. Sci. Appl. 6, 1-10 (2017).

6. D. Pérez, I. Gasulla, and J. Capmany, "Toward Programmable Microwave Photonics Processors," J. Light. Technol. 36, 519-532 (2018).

7. A. Peruzzo, A. Laing, A. Politi, T. Rudolph, and J. L. O'Brien, "Multimode quantum interference of photons in multiport integrated devices," Nat. Commun. 2, 224-226 (2011).

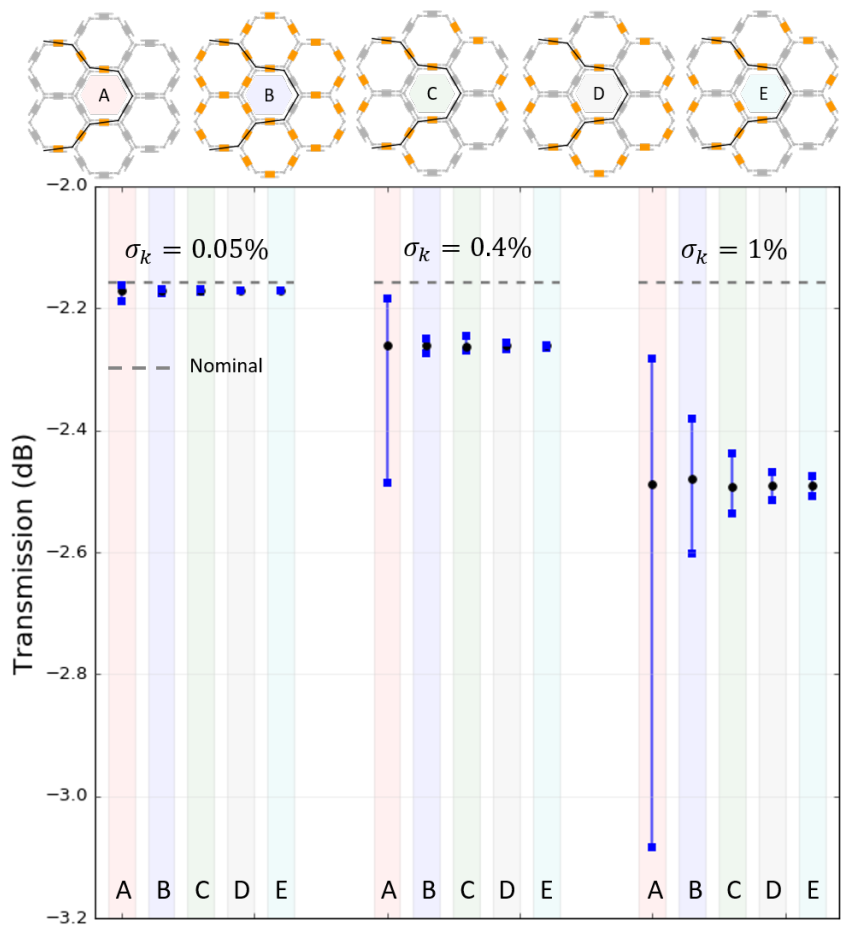

Fig. 9. Transmission spread analysis of a single path (with $\left.\Delta L=8 L_{u}\right)$ for different biasing schemes for $\sigma_{k}=$ $0.05 \%, 0.4 \%, 1.0 \%$.

8. B. J. Metcalf, N. Thomas-Peter, J. B. Spring, D. Kundys, M. A. Broome, P. C. Humphreys, X. M. Jin, M. Barbieri, W. Steven Kolthammer, J. C. Gates, B. J. Smith, N. K. Langford, P. G. Smith, and I. A. Walmsley, "Multiphoton quantum interference in a multiport integrated photonic device," Nat. Commun. 4, 1356-1357 (2013).

9. D. A. B. Miller, "Self-aligning universal beam coupler," Opt. Express 21, 6360 (2013).

10. D. A. B. Miller, "Self-configuring universal linear optical component [Invited]," Photonics Res. 1, 1 (2013).

11. J. Carolan, C. Harrold, C. Sparrow, E. Martín-López, N. J. Russell, J. W. Silverstone, P. J. Shadbolt, N. Matsuda, M. Oguma, M. Itoh, G. D. Marshall, M. G. Thompson, J. C. Matthews, T. Hashimoto, J. L. O'Brien, and A. Laing, "Universal linear optics," Science. 349, 711-716 (2015).

12. D. A. B. Miller, "Perfect optics with imperfect components," Optica. 2, $747(2015)$

13. N. C. Harris, G. R. Steinbrecher, M. Prabhu, Y. Lahini, J. Mower, D. Bunandar, C. Chen, F. N. Wong, T. Baehr-Jones, M. Hochberg, S. Lloyd, and D. Englund, "Quantum transport simulations in a programmable nanophotonic processor," Nat. Photonics 11, 447-452 (2017).

14. M. Reck, A. Zeilinger, H. J. Bernstein, and P. Bertani, "Experimental Realization of Any Discrete Unitary Operator," Phys. Rev. Lett. 73, 58-61 (1994).

15. N. C. Harris, J. Carolan, D. Bunandar, M. Prabhu, M. Hochberg, T. Baehr-Jones, M. L. Fanto, A. M. Smith, C. C. Tison, P. M. Alsing, and D. Englund, "Linear programmable nanophotonic processors," Optica. 5, 1623 (2018).

16. W. R. Clements, P. C. Humphreys, B. J. Metcalf, W. S. Kolthammer, and I. A. Walsmley, "Optimal design for universal multiport interferometers," Optica. 3, 1460 (2016).

17. D. Perez, I. Gasulla, F. J. Fraile, L. Crudgington, D. J. Thomson, A. Z. Khokhar, K. Li, W. Cao, G. Z. Mashanovich, and J. Capmany, "Silicon Photonics Rectangular Universal Interferometer," Laser Photonics Rev. 11 (2017).

18. S. Pai, B. Bartlett, O. Solgaard, and D. A. Miller, "Matrix Optimization on Universal Unitary Photonic Devices," Phys. Rev. Appl. 11, 1 (2019). 
19. D. Pérez, I. Gasulla, J. Capmany, and R. A. Soref, "Reconfigurable lattice mesh designs for programmable photonic processors," Opt. Express 24, 12093 (2016).

20. D. Pérez, I. Gasulla, L. Crudgington, D. J. Thomson, A. Z. Khokhar, K. Li, W. Cao, G. Z. Mashanovich, and J. Capmany, "Multipurpose silicon photonics signal processor core," Nat. Commun. 8, 1-9 (2017).

21. D. Pérez, I. Gasulla, and J. Capmany, "Programmable multifunctional integrated nanophotonics," Nanophotonics. 7, 1351-1371 (2018).

22. D. Pérez and J. Capmany, "Scalable analysis for arbitrary photonic integrated waveguide meshes," Optica. 6, 19 (2019).

23. A. Li, T. Van Vaerenbergh, P. De Heyn, P. Bienstman, and W. Bogaerts, "Backscattering in silicon microring resonators: A quantitative analysis," Laser Photonics Rev. 10, 420-431 (2016).

24. W. Bogaerts, M. Fiers, M. Sivilotti, and P. Dumon, "The IPKISS photonic design framework," 2016 Opt. Fiber Commun. Conf. Exhib. OFC 2016 pp. 1-3 (2016). 